\title{
Interaction between mechanosorptive and viscoelastic response of wood at high humidity level
}

\author{
C. Montero ${ }^{1, a}$, J. Gril $^{1}$, and B.Clair ${ }^{1}$
}

Laboratoire de Mécanique et Génie Civil, Université Montpellier 2, CNRS, 34090 Montpellier, France

\begin{abstract}
To clarify the contribution of mechanosorptive effect and compliance evolution according to moisture content during adsorption under load creep tests on longitudinal spruce specimens in variable environment were performed. By comparing different loading history we observe that creep increase during the first adsorption (++ effect) mostly results from the increase of compliance induced by higher moisture content. We also discuss the viscoelasticity and mechanosorptive interaction during creep.
\end{abstract}

\section{Introduction}

Since the first-published work in the 50's the time dependent behaviour raised an increasing interest in wood material research. Creep research is critical for in timber structures where the prediction of total deflection is necessary for durable structural design. The first approach to quantitative description of mechanosorptive creep was made by Ranta-Maunus [1] in 1975 based on plywood experimental results. He defined "hydroviscoelastic constants" to quantify the evolution of elastic compliance $J$ induced by change of moisture content $w$. Three notations has been created representing the slope $d J / d w$ during first adsorption " $a^{++}$", any subsequent desorption " $a^{-}$" or adsorption " $a^{+}$".

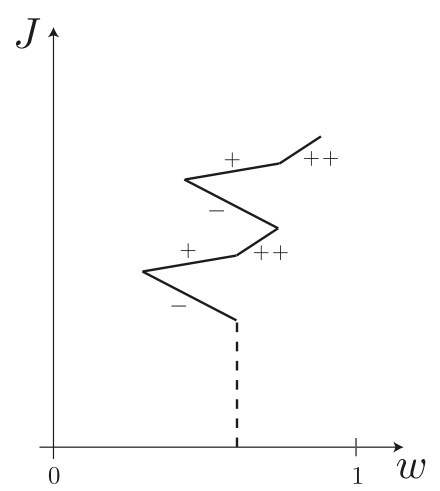

Figure 1. Hypothetical plot of compliance $\mathrm{J}$ against moisture content w (based on Ranta-Maunus description). Dashed line correspond to elastic compliance.

In 1984 according to new experimental results Hunt [2] observed that these constants depend on strain and suggested a limit to the amount of mechanosorptive creep approached asymptotically by repeated humidity cycles.

a e-mail: montero@lmgc.univ-montp2.fr 
In this study we propose to clarify the contribution of mechanosorptive effect and compliance evolution according to moisture content in the "++" effect. We also discuss on the viscoelasticity and mechanosorptive creep interaction.

\section{Material and methods}

\subsection{Wood material}

Matched spruce specimens were used in this study. Specimens have been $100 \mathrm{~mm}$ in longitudinal direction, $10 \mathrm{~mm}$ radial and $2 \mathrm{~mm}$ tangential directions. This geometry have been made to avoid multi-layer composition in specimens induced by annual rings which can produce discontinuity of strain profile with the sollitation. The average density have been determined $\rho_{\text {ref }}=0.48$ (at $\mathrm{T}=24^{\circ} \mathrm{C}, \mathrm{RH}=58 \%$ ). Preliminary measurements have been performed by X-ray diffraction. The mean microfibril angle has been measured before creep test as $16^{\circ}$ and the grain angle as $1^{\circ}$.

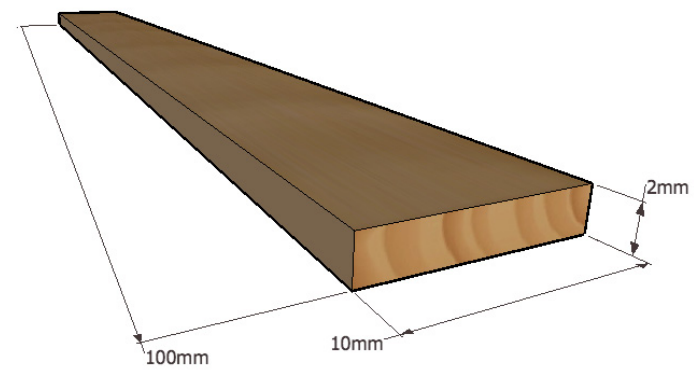

Figure 2. Illustration of specimen geometry and resulting annual rings distribution

\subsection{Creep equipment and measurements}

Four-point bending devices with $40 \mathrm{~mm}$ inner span and $80 \mathrm{~mm}$ outer span were used. Serial arrangement by pair of specimens has been done to increase repeatability in same environment conditions. Two sets of 4 specimens ( $i$-index) have been placed in controlled environment produced by a climatic chamber. Two specimens were kept unloaded for free swelling and shrinkage determination ( $j$-index). On each of these specimens strain $\varepsilon^{+}$was measured on tensile face and $\varepsilon^{-}$on compression face using electric strain gages.

Three specimens were weighted occasionally for moisture content estimation $w$.

\subsection{Data analysis}

In those bending tests the strain evolution is quantified by calculating mean strain $\langle\varepsilon\rangle$ and differential strain $\Delta \varepsilon$ for each sample.

$$
<\varepsilon>=\frac{\varepsilon^{+}+\varepsilon^{-}}{2} \quad \Delta \varepsilon=\frac{\varepsilon^{+}-\varepsilon^{-}}{2}
$$

The mean strain reveals hygromechanical processes occurring during sorption (as swelling and shrinkage). The differential strain reveals sample deflection.

A preliminary acclimatizing phase $(\mathrm{t}=0-360 \mathrm{~h})$ without specimen loading have been performed to clean out stress history of specimens and improve compliance calculations. The compliance have been calculated for each loaded specimen ( $i$-index) using the strain of corresponding unloaded specimen (eq. 2). A correction has been applied to eliminate small differences in density between specimens.

$$
J_{i}^{\text {corr }}=\frac{\rho_{i}}{\rho_{\text {ref }}} \frac{\left(\Delta \varepsilon_{i}-f_{i}\left(\Delta \varepsilon_{j}\right)\right)}{\sigma_{i}}
$$

A linear function have been established during acclimatizing phase with graphical analysis of $\Delta \varepsilon_{i}=$ $f_{i}\left(\Delta \varepsilon_{j}\right)$ to correct heterogeneities of specimen against sollicitation. The stress $\sigma_{i}$ was applied with lead mass and fixed below the elastic limit (around $8 \mathrm{MPa}$ ). 


\section{Results and discussion}

The complete environment history of the experiment and specimen response is describe in figure 3 . All these results have been made on longitudinal measurement. In this document only the creep part will be detailed.
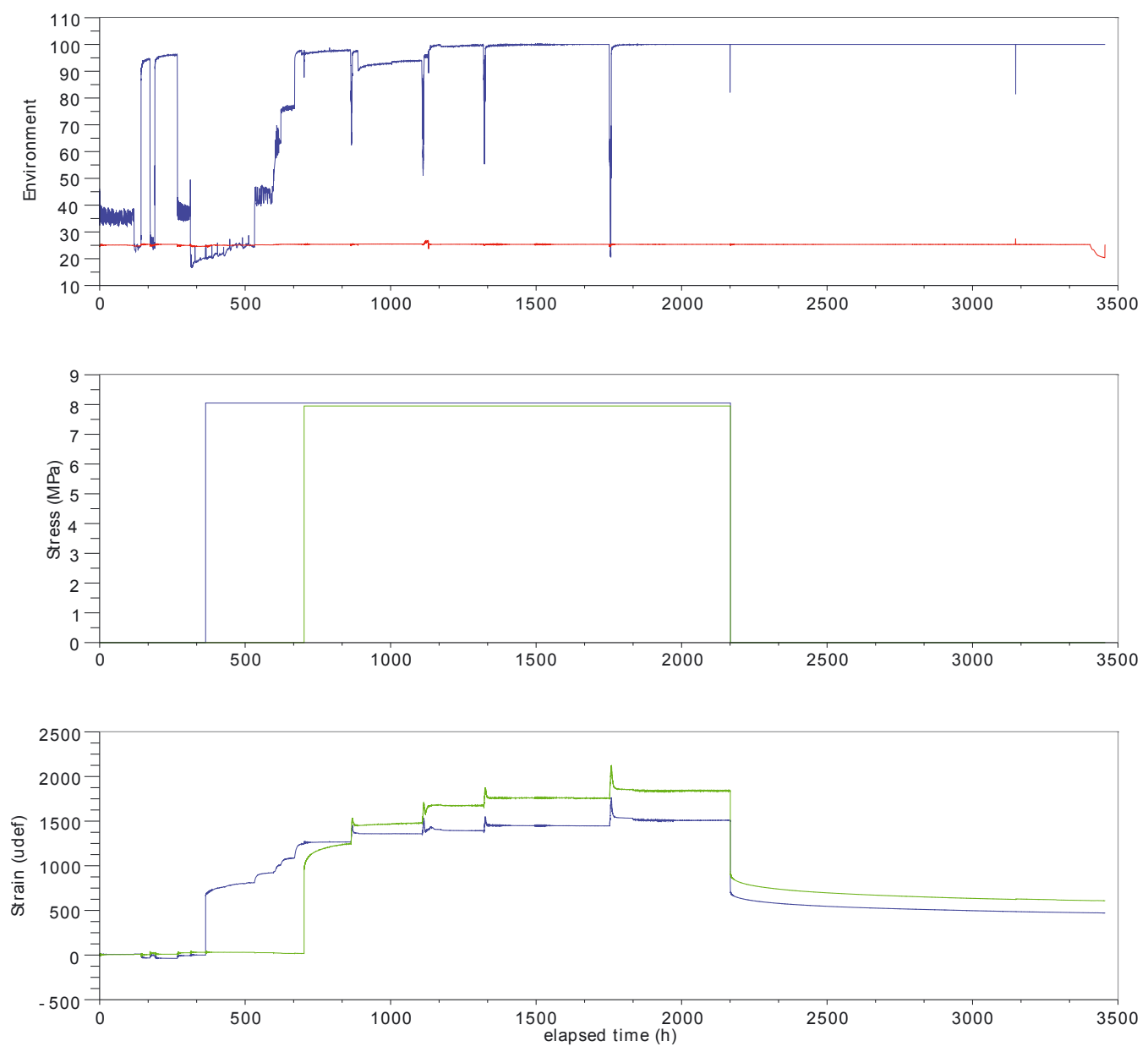

Figure 3. [Up] Experimental measure of the environment during the experiment (blue : relative humidity, red : temperature), [Middle] Mean stress history applied on specimens (blue : sorption A-set, green : viscous B-set), [Down] Mean strain history measured on specimens (blue : sorption A-set, green : viscous B-set).

\subsection{Moisture content influence on viscoelastic creep}

Constant environment periods at low and high relative humidity level have been used to quantify the influence of moisture content on viscoelastic creep. 


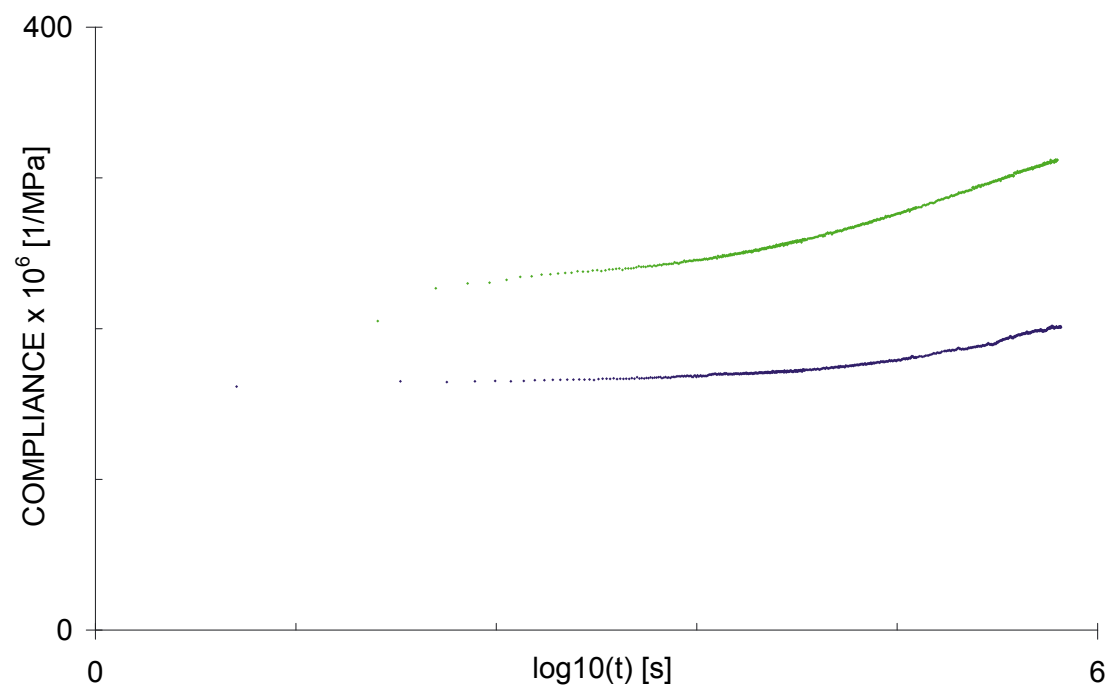

Figure 4. Comparison between compliance at low (blue) and high (green) moisture content against elapsed time of loading

In figure 4 the set $(\mathrm{A})$ maintained in dry state $(w \simeq 5 \%)$ presents as expected a lower elastic compliance than set $(\mathrm{B})$ at wet state $(w \simeq 27 \%)$.

\subsection{Mecanosorptive influence on viscoelastic response}

In the previous section the viscoelastic response have been analyzed near fiber saturated point $(w \simeq$ $27 \%$ ). A drying and a subsequent wetting of those specimens creates an strain increase. This phenomenon is called mechanosorptive creep.

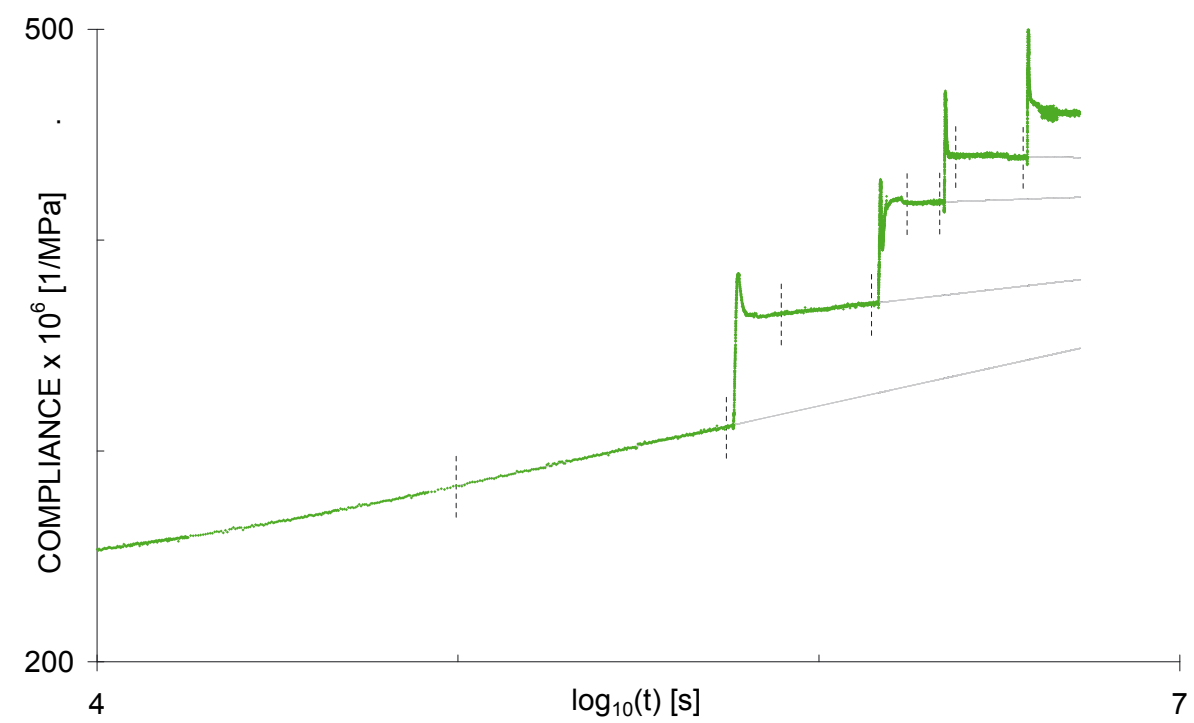

Figure 5. Compliance evolution at high moisture content of B-set specimens with (green) and linear compliance extrapolation after creep period (gray). 
Each creep set of constant humidity period have been linearly extrapolated from a suitable time range to determine the slope of mean compliance.

\begin{tabular}{|c|c|c|c|}
\hline Creep period & Log time range & $\begin{array}{c}\text { Log slope at the end } \\
\text { of creep period } \\
\mathbf{x 1 0}\left[\mathbf{M P a}^{-1}\right]\end{array}$ & $\begin{array}{c}\text { Change in log } \\
\text { slope } \\
\mathbf{x 1 0 ^ { 6 }}\left[\mathbf{M P a}^{-1}\right]\end{array}$ \\
\hline Initial & 0.75 & 35 & \\
\hline 1st cycle & 0.25 & 19.3 & -15.7 \\
\hline 2nd cycle & 0.09 & 6 & -13.3 \\
\hline 3rd cycle & 0.19 & -3.6 & -9.6 \\
\hline
\end{tabular}

Table 1. Slope evolution for set (B) specimens

This changes in slope indicates that viscoelastic creep depends on mecanosorptive creep accumulation indicating an interaction between both phenomenons. This result confirm Hanhijarvi's results on the influence between the viscoelastic response and mechanosorption [3]. It also seems to exhaust the viscoelastic response of material.

\subsection{Influence of moisture content at loading on mechanical response}

By loading the specimens at different moment of environment history we have studied the interaction between viscoelastic creep and mechanosorptive response.

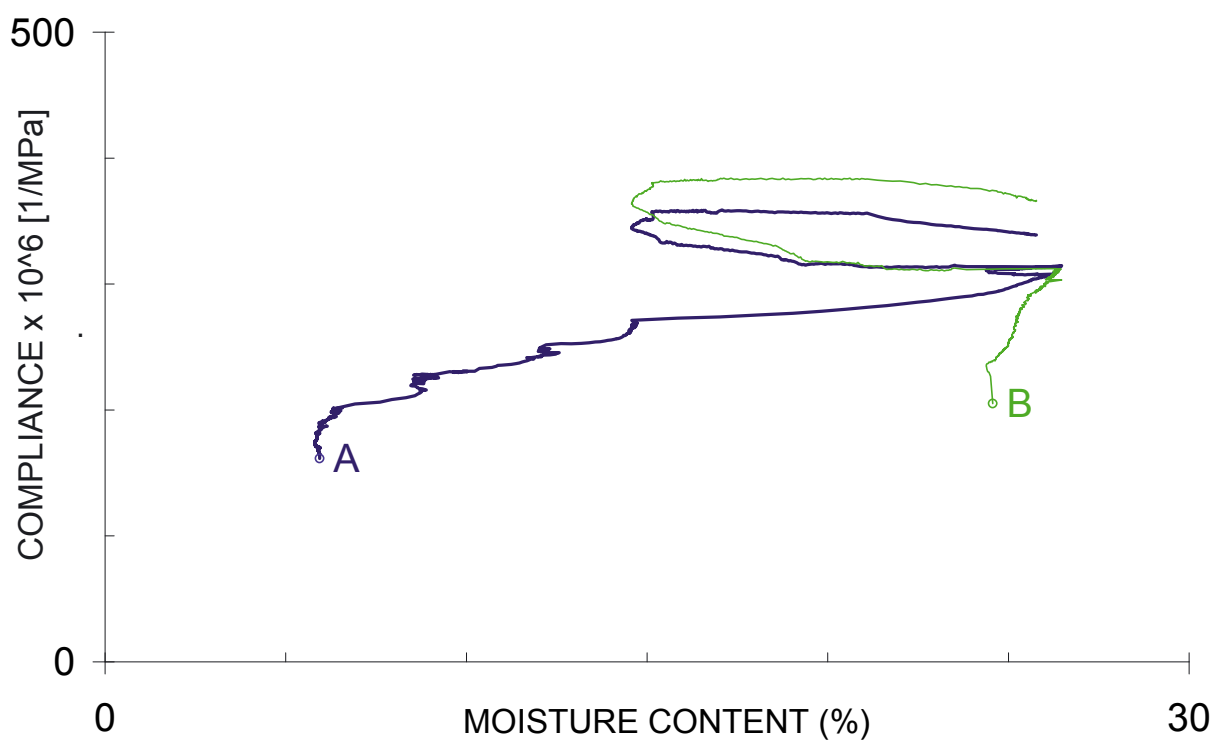

Figure 6. Compliance evolution for different loading history with one sorption cycle against estimated moisture content (blue : mean compliance of set A loaded at dry state, green : mean compliance of set B loaded at wet state)

The specimens (A) reach the deformation level of the specimens (B) suggesting that the "++" effect mostly results from the increase of compliance due to a higher moisture content. This observation supports the assumption that the mechano-sorptive effect does not occur significantly during adsorption 
at high humidity. In subsequent sorption cycles both sets (A) and (B) exhibit mechanosorption. In set (B), neither one week of wet creep nor subsequent sorption cycles could exhaust mechano-sorption. This results suggests that mechanosorption and pure creep are two separate phenomena.

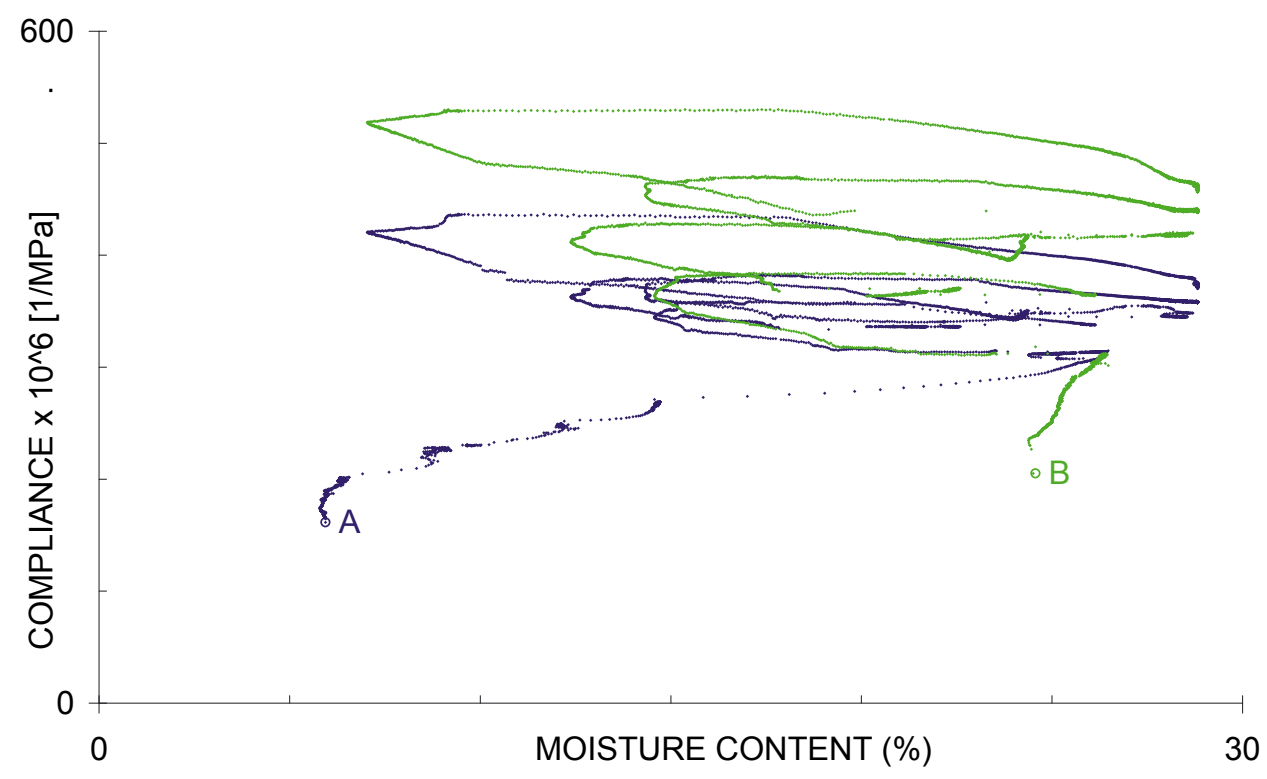

Figure 7. Compliance evolution for different loading history with many sorption cycle against estimated moisture content (blue : mean compliance of set A loaded at dry state, green : mean compliance of set B loaded at wet state)

However the effect of these dry-wet cycles is much less pronounced with set (A), which reached an apparent stable-state creep limit whatever the amplitude of dry-wet cycles. This indicates that the mechanosorptive response interacts in a complex manner with pure creep and that it cannot be simply described by an additive strain component. These results evidence the influence of moisture content history during loading and give useful indications on the interaction between mechanosorptive effect and viscoelasticity.

\section{References}

1. A. Ranta-Maunus, Wood science and technology 9/3, 189 (1975)

2. D.G. Hunt, Journal of materials science 19/5, 1456 (1984)

3. A. Hanhijärvi, D.G. Hunt, Wood science and technology 32/1, 57 (1998) 